\title{
Small Bowel Obstruction Caused by Blood Clots: A Rare Complication of Peptic Ulcer
}

\author{
Naoto Mizumura ${ }^{\mathrm{a}, \mathrm{b}}$, Atsuo Imagawa ${ }^{\mathrm{a}}$, Masayasu Kawasaki ${ }^{\mathrm{a}}$, Satoshi Okumura ${ }^{\mathrm{a}}$, \\ Sho Toyoda ${ }^{\mathrm{a}}$, Masao Ogawa ${ }^{\mathrm{a}}$
}

\begin{abstract}
Small bowel obstruction (SBO) caused by blood clots is an extremely rare complication of peptic ulcer. A 20-year-old woman presented to our hospital with coffee ground vomitus and diffuse abdominal tenderness. Computed tomography (CT) findings led us to the diagnosis of complete SBO caused by blood clots as a result of bleeding from a gastric ulcer. We successfully treated the patient by dissolution and evacuation therapy using a long intestinal tube, and no surgery was required. Peptic ulcer may cause occlusive intraluminal hematoma. In such cases, CT findings and long tube therapy may help avoid surgery.
\end{abstract}

Keywords: Blood clots; Intraluminal hematoma; Long intestinal tube; Peptic ulcer; Small bowel obstruction

\section{Introduction}

Peptic ulcer is a common disease worldwide. Bleeding, perforation, penetration, and gastric outlet obstruction are common complications of peptic ulcer, whereas small bowel obstruction (SBO) caused by blood clots is extremely rare. Although only two cases, including stress-induced duodenal bleeding [1] and warfarin-induced gastric bleeding [2], have been reported thus far, both the cases were diagnosed with surgery $[1,2]$. To our knowledge, there have been no previous reports of non-operative management using a long tube. Here, we report a case of occlusive intraluminal hematoma, wherein computed tomography (CT) findings and long tube therapy helped avoid surgery.

\section{Case Report}

A 20-year-old woman with no remarkable medical history

Manuscript accepted for publication September 17, 2015

aDepartment of Surgery, Bellland General Hospital, 500-3 Higashiyama, Naka-ku, Sakai-city, Osaka 599-8247, Japan

${ }^{\mathrm{b} C}$ Corresponding Author: Naoto Mizumura, Department of Surgery, Bellland General Hospital, 500-3 Higashiyama, Naka-ku, Sakai-city, Osaka 599-8247, Japan. Email: n_mizumura@seichokai.or.jp

doi: http://dx.doi.org/10.14740/jmc2298w presented to our hospital because of several episodes of coffee ground vomitus lasting half a day. She had epigastric pain and black stool for 1 week. Her heart rate and blood pressure were 131 beats/min and 119/74 $\mathrm{mm} \mathrm{Hg}$, respectively. Physical examination revealed diffuse abdominal tenderness without peritoneal signs. Laboratory findings were as follows: white blood cell count, 8,300 cells $/ \mathrm{mm}^{3}$; hemoglobin level, $15 \mathrm{mg} /$ dL; blood urea nitrogen level, $19.6 \mathrm{mg} / \mathrm{dL}$; creatinine level, $1.03 \mathrm{mg} / \mathrm{dL}$; and C-reactive protein level, $5.94 \mathrm{mg} / \mathrm{L}$. Her blood coagulation tests, liver function, blood gas analysis, and common tumor marker levels were normal. On admission, contrast-enhanced CT revealed submucosal thickening of the gastric antrum and a site of obstruction in the ileum with dilation of the proximal small bowel (Fig. 1). Although we initially considered that the patient presented with gastrointestinal necrosis, a comma-shaped high-density mass (approximately $60 \times 26 \mathrm{~mm}$ ) led us to the diagnosis of complete SBO caused by blood clots as a result of bleeding from a gastric ulcer. On admission, under fluoroscopy, a long intestinal tube was placed in the terminal ileum with suction and irrigation using warm saline. Approximately $1 \mathrm{~L}$ of dark green fluid and blood clots were evacuated. Consequently, the obstruction was relieved, and the contrast medium reached the colon (Fig. 2). This procedure lasted approximately $60 \mathrm{~min}$ without complications. Because follow-up CT showed neither the mass nor the dilated small bowel, the tube was removed on hospital day 2. On hospital day 3, endoscopy revealed submucosal thickening with a deep ulcer (Forrest III lesion) in the gastric antrum (Fig. 3). After administration of intravenous omeprazole $20 \mathrm{mg}$ twice a day and fasting for 6 days, she recovered and was discharged on hospital day 9. Capsule endoscopy and follow-up endoscopy performed 1 month after discharge showed no evidence of lesions or blood clots in the small bowel. The ulcer had healed, and biopsies of this region showed an unremarkable pathology. She tested negative for Helicobacter pylori.

\section{Discussion}

This case highlighted two important issues. First, blood clots as a result of bleeding from the peptic ulcer caused complete $\mathrm{SBO}$, and CT is useful as a diagnostic modality for such cases. Siddiky and Gupta [1] reported a case of SBO caused by jejunal 

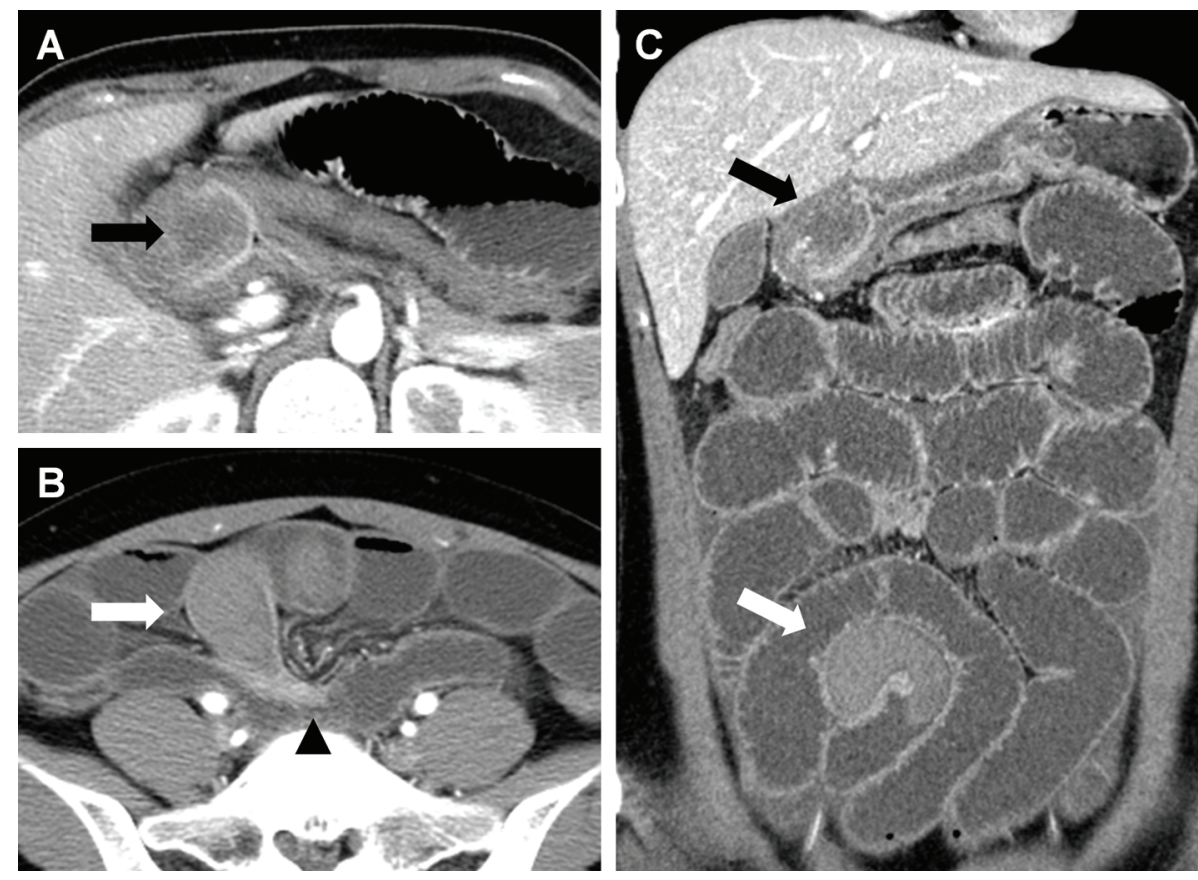

Figure 1. (A, B) Axial computed tomography. (C) Coronal computed tomography. Computed tomography showing submucosal thickening of the gastric antrum (black arrows), a comma-shaped high-density mass (white arrows) in the ileum, and dilated small bowel loops with a transitional zone (black arrow head), but absence of intestinal ischemia.

impaction of blood clots observed in the duodenum by endoscopy 3 days prior. Similarly, our patient possibly had a gastric ulcer with blood clots in the gastrointestinal tract, and ileal impaction of the blood clots conclusively caused complete SBO. The most common site of impaction of gastrointestinal foreign bodies is the ileum, which is the narrowest part of the bowel $[3,4]$. Enteroscopy, although useful to detect hematomas in the

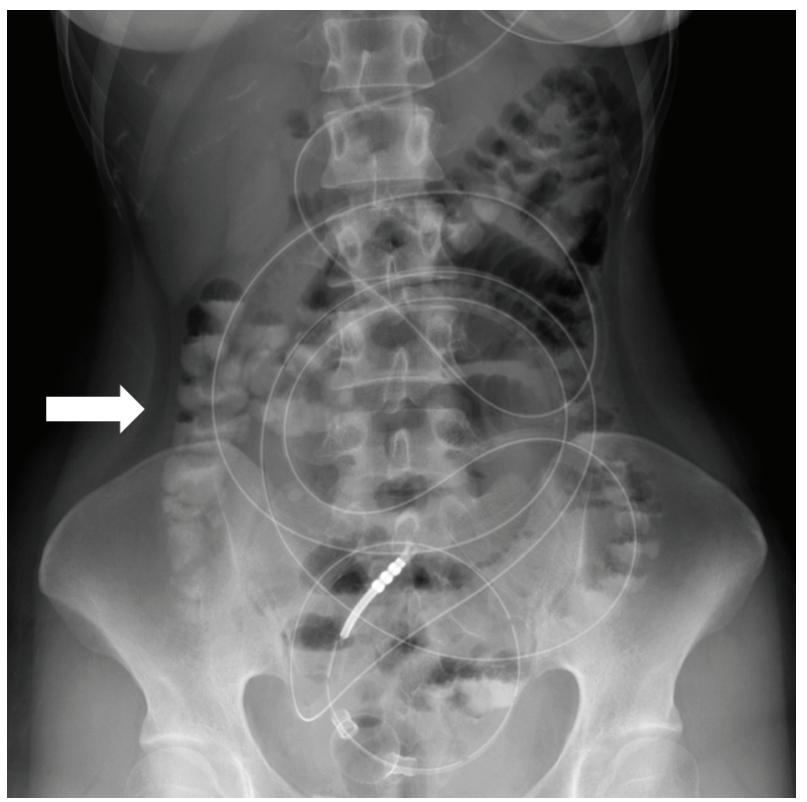

Figure 2. An abdominal radiograph showing the contrast medium in the colon (white arrow). small bowel, is difficult to perform in an emergency setting. Therefore, occlusive intraluminal hematoma is diagnosed only with surgery $[1,2]$. We initially considered the mass to be a bezoar or gallstone [3]. However, the mass had a uniform high density (58 Hounsfield units) without internal gas and was not round like a gallstone but was rather loop-shaped located along the small bowel, which led to the diagnosis of a hematoma.

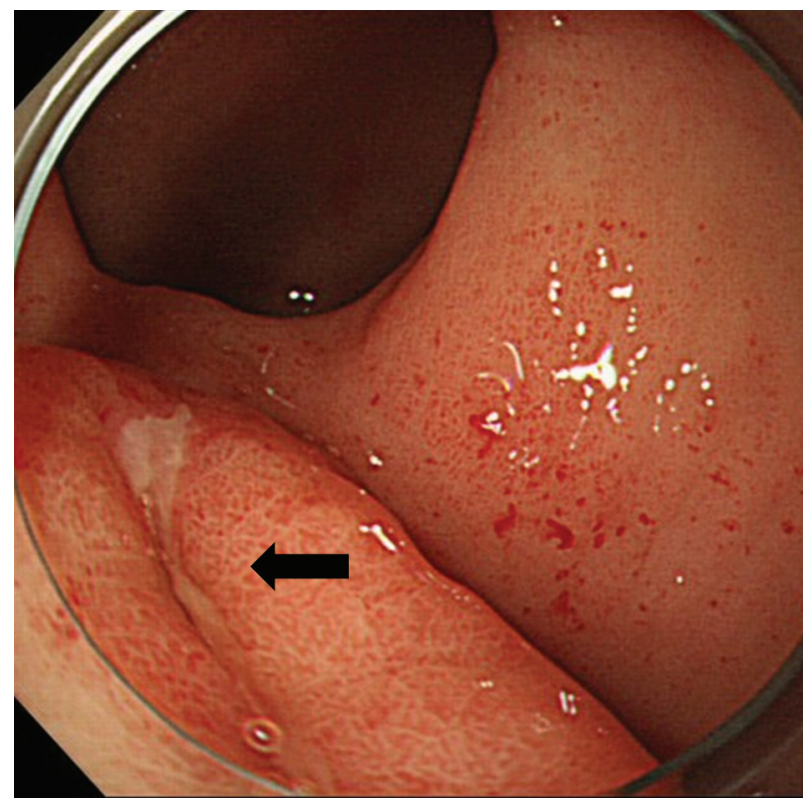

Figure 3. Endoscopic findings showing submucosal thickening with a deep ulcer (black arrow). 
Additionally, because there was no circumferential bowel wall thickening or mucosal fold thickening [5], we determined that the mass was not an intramural hematoma but rather an intraluminal hematoma.

Second, non-operative management using a long tube is useful to avoid surgery. We initiated treatment of the SBO before performing endoscopy for the gastrointestinal bleeding (Glasgow Blatchford score: 4) [6]. In the absence of clinical, laboratory, or radiologic findings of bowel ischemia, patients with SBO undergo non-operative management [7]. In patients with bezoars, dissolution therapy with cola through a long tube has been reported [8]. Similarly, in patients with occlusive intraluminal hematoma, long tube therapy (including waiting for spontaneous absorption or passage, dissolution with warm saline, and evacuation by suction) may relieve the obstruction.

\section{Conclusion}

In patients with hematemesis, if patients simultaneously present with SBO, we generally consider the possibility of gastrointestinal necrosis $[9,10]$. Although rare, peptic ulcer may cause occlusive intraluminal hematoma. In such cases, CT findings and long tube therapy may help avoid surgery.

\section{Grant Support}

None.

\section{Conflict of Interest}

None.

\section{References}

1. Siddiky A, Gupta P. Proximal small bowel obstruction caused by a massive intraluminal thrombus from a stress ulcer. J Surg Case Rep. 2012;2012(1):6.

2. Maimon N, Penner T, Demajo W. Warfarin-induced gastric bleeding and intestinal obstruction. Eur J Intern Med. 2006;17(3):211-212.

3. Ayantunde AA, Agrawal A. Gallstone ileus: diagnosis and management. World J Surg. 2007;31(6):1292-1297.

4. Velitchkov NG, Grigorov GI, Losanoff JE, Kjossev KT. Ingested foreign bodies of the gastrointestinal tract: retrospective analysis of 542 cases. World J Surg. 1996;20(8):1001-1005.

5. Abbas MA, Collins JM, Olden KW. Spontaneous intramural small-bowel hematoma: imaging findings and outcome. AJR Am J Roentgenol. 2002;179(6):1389-1394.

6. Blatchford O, Murray WR, Blatchford M. A risk score to predict need for treatment for upper-gastrointestinal haemorrhage. Lancet. 2000;356(9238):1318-1321.

7. Maung AA, Johnson DC, Piper GL, Barbosa RR, Rowell SE, Bokhari F, Collins JN, et al. Evaluation and management of small-bowel obstruction: an Eastern Association for the Surgery of Trauma practice management guideline. J Trauma Acute Care Surg. 2012;73(5 Suppl 4):S362-369.

8. Zheng YX, Prasoon P, Chen Y, Hu L, Chen L. "Sandwich" treatment for diospyrobezoar intestinal obstruction: a case report. World J Gastroenterol. 2014;20(48):18503-18506.

9. Bou-Abdallah JZ, Murthy UK, Mehta N, Prasad HN, Kaul V. Coffee grounds emesis: not just an upper GI bleed. J Emerg Med. 2012;43(1):44-46.

10. Moissinac K, Ponnampalam J, Chong Se To B. Haematemesis resulting from ischaemic strangulating intestinal obstruction. Eur J Emerg Med. 2000;7(4):297-299. 